\title{
El Estado mexicano respecto al derecho humano a la protección de la salud
}

\author{
The Mexican State regarding the human right to health \\ protection
}

\section{Gustavo Aguilera Izaguirre}

Doctor en Derecho por la Universidad de Salamanca, España; máster en Estado de Derecho y Corrupción por la Universidad de Salamanca, España; especializado en Ciencias Políticas y Estudios Constitucionales por el Centro de Estudios Políticos y Constitucionales del Ministerio de la Presidencia de España; docente investigador, Facultad de Derecho de la Universidad Autónoma del Estado de México. Correo electrónico: gusyr37@hotmail.com

\section{Resumen}

El presente artículo tiene como finalidad la evolución y el análisis del derecho a la protección de la salud como derecho humano en relación con el contexto mexicano, el desarrollo de políticas públicas y funcionamiento de su Sistema de Salud a lo largo de los años a efecto de garantizar el acceso igual y universal a los servicios de salud. Una breve referencia a la problemática acerca de la naturaleza jurídica y exigibilidad de los derechos económicos, sociales y culturales en un contexto general para después abordar el tema en el ordenamiento jurídico mexicano.

Palabras clave: Derecho humano, protección a la salud, políticas públicas, mecanismos de exigibilidad.

Abstract

This article presents the evolution and analysis of the right to health protection as a human right in relation to the Mexican context, the development of public policies and the performance of the Health System during the past years in order to guarantee the equal and universal access. A brief review regarding the problematic around the legal nature and the enforceability of the economic, social and cultural rights in a general context and then address the issue to the Mexican legal order.

Keywords: Human right, health protection, public policies, enforceability mechanisms.

\section{Résumé}

Cet article vise à l'élaboration et à l'analyse du droit à la protection de la santé comme un droit humain en relation avec le contexte mexicain, le développement des politiques publiques et le fonctionnement du système de santé au cours des années à effet assurer un accès égal et universel aux services de santé. Une brève référence aux problèmes concernant la nature juridique et le caractère exécutoire des droits économiques, sociaux et culturels dans un contexte général puis aborder la question dans le système juridique mexicain.

Mots-clés: droits de l'homme, la protection de la santé, les politiques publiques, les mécanismes d'application. 



\section{El Estado mexicano respecto al derecho humano a la protección de la salud}

Gustavo Aguilera Izaguirre

\section{DERECHOS HUMANOS}

Históricamente los derechos humanos son el resultado de la lucha de ideologías impulsadas por movimientos sociales, tales como: la Revolución Francesa y la independización de las colonias inglesas en Norteamérica, por mencionar algunos. Estas ideologías encontraban su base fundamental en ideas como la libertad, igualdad y dignidad que de cierta manera se contraponían con los regímenes absolutistas (Peces-Barba, 1987). A partir de este momento la actuación del Estado se ve limitada y el límite estaba constituido por los derechos de los individuos. Derechos que se plasmaban en los ordenamientos jurídicos a efecto de constituir un parámetro tangible para la correcta delimitación de las potestades del Estado.

La corriente positivista adquirió fuerza con Kelsen, aquello que no estaba plasmado en la norma no constituía un objeto de Derecho y esto coadyuvó a la confusión de los derechos humanos con las técnicas jurídicas contenidas en los ordenamientos legales, o bien a una correlatividad que justifica la existencia de un derecho a partir de la existencia de un mecanismo jurídico de exigibilidad.

La cuestión de los derechos humanos en el contexto actual ha tomado un giro en cuanto a su concepción, se ha abordado de diferentes perspectivas, pero la más imponente es sin duda la idea de que los derechos humanos se deben de tomar como la razón que justifica la normatividad que protege determinado bien y no a la inversa (Laporta, 1987). 
Entonces debemos entender que la positivización de los derechos es el último paso del eslabón y los cimientos morales y justificación se encuentran eminentemente en el componente racional que protege a la persona por el simple hecho de pertenecer a la especie humana en lo que respecta a su dignidad humana, libertad e igualdad (Peces-Barba, 1987).

Los derechos humanos poseen ciertas características, son universales en tanto que no distinguen entre condiciones económicas, sociales, culturales, étnicas, de género, entre otras y pertenecen al ser humano por esencia, y cualquier negación de estos en atención a esas diferencias representa una clara violación.

Son inalienables, puesto que no son renunciables y representan un límite en la autonomía de la voluntad del propio titular y de las demás personas, ya que este no puede disponer de estos como cualquier otro bien y no son objeto de cancelación bajo ninguna circunstancia.

Los derechos coexisten, tanto los derechos individuales como colectivos representan un todo para su titular y hay cierta codependencia en el debido goce de unos y otros. Por ejemplo, una persona que pretende ejercer el derecho de propiedad sobre otro debe primero disponer de un mínimo de bienes que le aseguren una vida digna. Es precisamente la razón por la cual el Estado ha dejado atrás la concepción individualista de los derechos humanos y ha dejado de limitar su actuación como simple vigilante para participar como proveedor y guardián de todos y cada uno de los derechos de las personas.

La comunidad internacional ha sido partícipe en la adquisición de compromisos por parte de los Estados, a efecto de planear la acción gubernamental, es decir, implementar criterios de racionalidad en el desarrollo de políticas públicas e incluso elaboraciones de presupuestos que giren en torno a la protección y aseguramiento de los derechos humanos (Oficina del Alto Comisionado de las Naciones Unidas, 2010).

Para efectos únicamente enunciativos en México la Suprema Corte de Justicia define a los derechos humanos como:

Prerrogativas mínimas que todo miembro de la especie humana, por su propia naturaleza debe gozar, y cuyo respeto y observancia deben ser garantizados por el Estado en todo tiempo y lugar, pues a través de ellas se concretan las exigencias de la dignidad humana (Corte, 2013).

\section{DERECHO A LA PROTECCIÓN DE LA SALUD COMO DERECHO HUMANO EN EL CONTEXTO INTERNACIONAL}

El desarrollo de los derechos humanos es un fenómeno histórico y dinámico, las necesidades del hombre con el transcurso del tiempo adquieren importancia indispensable para su desarrollo y no por eso dejan de existir las ya establecidas en el catálogo de derechos. Si bien los derechos individuales y fundamentales del hombre han sido reconocidos desde la Revolución Francesa de 1789, sustentados como el límite de actuación de las autoridades estatales erigiéndose de esta manera 
el Estado de Derecho, a principios del siglo XX se desarrolla el derecho social como aquellos derechos que van encaminados a sectores desprotegidos de la sociedad en pro de la igualdad y equilibrio de esta. Luego, se consideran como una extensión de los primeros derechos, puesto que significan expectativas mínimas de satisfacción de necesidades a efecto de poder hacer uso y goce pleno de los demás derechos (Moctezuma, 2000).

Los derechos sociales emergen como respuesta a las políticas públicas liberales desplazadas en la Revolución Francesa de 1789. El sistema individualista liberal laissez faire, faire passer acentuaba las desigualdades entre las diferentes clases sociales, dotando de privilegios a la clase burguesa con base en su estatus social y poder adquisitivo.

La corriente del derecho social va encaminada a equilibrar las disparidades naturales de una sociedad de mercado, haciendo énfasis en actividades que desarrolladas únicamente por particulares, en busca de su propio beneficio, harían a un lado el bienestar general, por lo que el Estado adquiere una obligación, tanto prestacional en el sentido de que debe garantizar, por lo menos el mínimo racional de esas necesidades, como de abstención en el sentido de que no las debe transgredir (Tapia \& Motta, 2005).

En este contexto de individualismo, la salud física se dejaba exclusivamente al gasto privado y se concebía como un bien dentro del mercado, la concepción del uso del servicio no era mediante parámetros de necesidad (como actualmente se pretende concebir) si no por capacidad de consumo. A partir de la Segunda Guerra Mundial con el surgimiento del Estado de bienestar en las comunidades europeas, el servicio a la salud y derecho a acceder a este de forma gratuita se otorga solo por tener la calidad de ciudadano o en ciertos casos solo por estar dentro del territorio (Mayer-Serra, 2007). En lo que respecta al derecho a la protección de la salud, este tiene su ubicación como derecho humano en la clasificación de los derechos económicos, sociales y culturales. La concepción en el ámbito internacional de estos derechos sin duda ha redirigido las actuaciones de los Estados en cuanto a políticas públicas encaminadas a la satisfacción de estos, ponderando los derechos sociales como aquellos derechos que anteceden el goce de otros derechos fundamentales y ubicándolos como parámetro para la medición del desarrollo de los Estados.

La problemática de la concepción del derecho a la protección de la salud como derecho humano gira en torno a su conceptualización y por lo tanto a su exigibilidad en determinado ordenamiento jurídico (Carbonell, 2012). En términos reales, la salud no es un bien cuantificable mediante parámetros exactos, es un estado que depende de diversas circunstancias aleatorias, controladas en cierta medida, pero no en su totalidad, por el Estado. Por tanto, el Estado tiene la obligación de garantizar en la medida de lo posible, racional y hasta donde sus recursos económicos alcancen, la protección de este derecho a los individuos sin distinción alguna de su condición social, económica, étnica, geográfica, sexo, entre otros. Este derecho adquiere una conducta de carácter prestacional como obligación del Estado aunado a la abstención 
de transgredirla, lo que se traduce en la obligación de incluir en las políticas públicas de salud la realización de objetivos encaminados a satisfacer los tres aspectos que representan la protección de la salud, a saber: el acceso a los servicios de asistencia médica, la salud pública y la asistencia social (Moctezuma, 2000).

Son diversas las organizaciones internacionales y los instrumentos jurídicos que contienen y garantizan la protección de la salud como derecho humano.

La Organización Mundial de la Salud (1946) define a la salud como: "La salud es un estado de completo bienestar físico, mental y social, y no solamente la ausencia de afecciones o enfermedades".

La Organización pondera este derecho como base fundamental para un correcto desarrollo de la persona en lo individual y colectivo, los Estados en la prestación de los servicios de salud tendrán que implementar tres características que representan de cierta manera un parámetro de exigibilidad, a saber son: la universalidad, equidad y calidad (Carbonell, 2012).

La declaración Universal de los Derechos del Hombre y del Ciudadano (1948) por otro lado lo define en su artículo 25 como un elemento fundamental de la vida digna:

Toda persona tiene derecho a un nivel de vida adecuado que le asegure, así como a su familia, la salud y el bienestar y en especial la alimentación, el vestido, la vivienda, la asistencia médica y los servicios sociales necesarios; tiene asimismo derecho a los seguros en caso de desempleo, enfermedad, invalidez, vejez $u$ otros casos de perdida de sus medios de subsistencia por circunstancias independientes de su voluntad.

En el Pacto Internacional de los Derechos Sociales, Económicos y Culturales (1966), los estados se comprometen a garantizar los derechos mediante la implementación de políticas públicas, instrumentación jurídica en sus ordenamientos para su protección y hasta donde sus recursos económicos alcancen, la piedra angular de estos derechos descansa en la igualdad que tienen los seres humanos a tener una vida digna. En su artículo 12 se encuentra el derecho a la salud de la siguiente manera:

Los Estados Partes en el presente Pacto reconocen el derecho de toda persona al disfrute del más alto nivel posible de salud física y mental. Entre las medidas que deberán adoptar los Estados Partes en el Pacto a fin de asegurar la plena efectividad de este derecho, figurarán las necesarias para:

a) La reducción de la mortalidad natal y de la mortalidad infantil, y el sano desarrollo de los niños;

b) El mejoramiento en todos sus aspectos de la higiene del trabajo y del medio ambiente;

c) La prevención y el tratamiento de las enfermedades epidémicas, endémicas, profesionales y de otra índole, y la lucha contra ellas; 
d) La creación de condiciones que aseguren a todos asistencia médica y servicios médicos en caso de enfermedad.

En la declaración de Alma Ata (1978) por otro lado se centra en la atención primaria de salud y la importancia para la protección de esta, retoma el concepto de salud como:

Estado de completo bienestar físico, mental y social, y no solamente la ausencia de afecciones o enfermedades, es un derecho humano fundamental y que el logro del grado más alto posible de salud es un objetivo social sumamente importante en todo el mundo, cuya realización exige la intervención de muchos otros sectores sociales y económicos, además del de la salud.

Insta a los gobiernos a dar especial atención a esta área, además de definir los servicios que comprende, los parámetros de la prestación de servicio de manera equitativa y reiterar la problemática de desigualdad así como la importancia de este derecho tanto individual como colectivo.

Otros ordenamientos internacionales como la Convención Internacional sobre la Eliminación de toda forma de Discriminación Racial (1965), Convención Interamericana contra toda forma de Discriminación e Intolerancia (OEA), Convención sobre la Eliminación de toda forma de Discriminación contra la Mujer (1979) garantizan que el Estado no trasgreda el disfrute y protección de los derechos por determinada condición del individuo.

En el 2000 la Asamblea General de la ONU desarrolló y sintetizó "Los Objetivos de Desarrollo del Milenio", donde 189 países se comprometen a la colaboración internacional, así como la implementación de sus políticas publicas encaminadas al desarrollo sustentable y la erradicación de la pobreza. En cuanto a la salud, los objetivos primordiales son la reducción de la tasa de mortalidad infantil, reducción de la tasa de mortalidad materna, combate contra el SIDA, malaria y otras enfermedades así como la protección y sostenibilidad del medio ambiente. La mayoría de los compromisos tiene como meta el presente año y han sido objeto de estudio y evaluación por el comité establecido también por la ONU, México es uno de los países que se unió al acuerdo y de cierta manera las últimas reformas hechas al sistema de salud y a la implementación de la protección de la misma se han visto impulsadas por la presión internacional en aras de desarrollo social (Lusitg, 2008).

\section{DERECHO A LA SALUD COMO DERECHO HUMANO EN MÉXICO}

En un principio debido a la influencia de España en la Colonia, la salud se concebía como una función de beneficencia a cargo de la iglesia, después de diversos movimientos y cambios sociales fue hasta la promulgación de las Leyes de 
Reforma en 1979 donde la Iglesia y el Estado se separan y el tema de salud pasa a ser responsabilidad única del Estado pero en un contexto muy individualista (Tapia \& Motta, 2005).

Es hasta la Constitución de 1917 cuando por primera vez se plasma la concepción del derecho social en favor de sectores de la sociedad sometidos a notables disparidades como resultado de las políticas públicas liberales del Porfiriato. Es así, como la clase obrera y campesina obtiene el reconocimiento de sus derechos en apartados constitucionales. En lo que respecta a la salud, los Constituyentes del 17, recogen el modelo de prestaciones de la política pública de Otto Von Bismarck (Alemania), donde se establecieron ciertas prerrogativas en favor de la clase trabajadora, tales como las pensiones, las compensaciones por riesgo en el trabajo, seguro de enfermedad, seguro de desempleo, es decir, prestaciones sociales que protegían al individuo de las situaciones desventajosas e injustas, ya fueran naturales o causadas por el hombre, mejor conocido como el welfare state (Chertorivski, 2012) ${ }^{1}$. Cabe mencionar que nuestros legisladores también retomaron las fallas que llevaron a este sistema al fracaso, el interés de Bismarck iba encaminado a los trabajadores de corte industrial, de esta manera se impulsaban los intereses capitalistas, pero a la vez se otorgaban privilegios al proletariado, sin embargo, muchas personas trabajadoras en otros sectores quedaron excluidas al no representar un incremento en el flujo capitalista (Baldwin, 1990).

De la misma manera se adopta en México el derecho a la salud y a la seguridad social como perteneciente a una clase específica, en otras palabras, el sector formal de la economía. De tal manera que no es un derecho para todos los mexicanos y se restringe a los trabajadores y sus familiares. Por lo tanto se inspira de esta manera la redacción de la fracción XXIX del apartado A del artículo 123 constitucional en el Título Sexto "Del Trabajo y la Previsión Social". En 1943 se crea el Instituto Mexicano del Seguro Social y posteriormente en 1960, como réplica del modelo de este, surge para trabajadores del Estado el Instituto de Seguridad Social al Servicio de los Trabajadores del Estado (ISSSTE) (Mayer-Serra, 2007).

En el transcurso de los años ochenta, el país se vio inmerso en una recesión laboral por lo que el modelo del sistema de salud era por demás inadecuado, dejando a muchos mexicanos sin acceso a los servicios de salud. En un esfuerzo político por cimentar las bases de un cambio en el sistema, durante el sexenio del presidente Miguel de la Madrid Hurtado en 1983 se reconoce el derecho a la protección de la salud en el artículo cuarto constitucional, quedando plasmado como derecho fundamental en la constitución de la siguiente manera: "Toda persona tiene derecho a la protección a la salud, la ley definirá las bases y modalidades para el acceso a los servicios de salud".

1 Entrevista realizada al ex secretario de Salud Salomón Chertorivski en el programa Espiral de la cadena Once TV México el 28/11/2012. 
Lo siguiente desató un número considerable de críticas, primero en relación a su posicionamiento con otros derechos de la misma índole, la mala técnica constitucional que no define la sustancia del derecho, no se expresa de manera explícita la cobertura universal, ni los parámetros de exigibilidad, según Miguel Carbonell lo que se plasmó fue un "mosaico de derechos" (Carbonell, 2012). Otra crítica importante es que el reconocimiento en letra de los derechos no significa su existencia y aplicabilidad en la realidad, derecho que no está contemplado en la erogación por parte del Estado, no es derecho (Mayer-Serra, 2007). No bastan entonces las buenas intenciones, es necesaria la implementación de políticas públicas, manejo eficiente de recursos, tanto materiales como humanos, e instrumentación jurídica que regule la calidad y actuación del Estado así como, en el caso de México y otros países de América Latina, un cambio sustancial y re organizacional en el Sistema de Salud que elimine las desigualdades latentes en los diversos sectores de la sociedad y hagan tangible la existencia del derecho. En los siguientes apartados se realizará un análisis de la evolución de las políticas públicas y el sistema de salud mexicano así como los esfuerzos gubernamentales y las deficiencias en las que se han incurrido en aras de la existencia y protección del derecho a la salud.

\section{EVOLUCIÓN DE POLÍTICAS PÚBLICAS DE SALUD EN MÉXICO}

\section{3-1983}

De acuerdo con Julio Frenk, ex secretario de salud durante el sexenio del presidente Vicente Fox, el año que marca la intervención del Estado en el ámbito de la salud es 1943, se crea el primer organismo de la administración pública estatal para dirigir el sector y surge con el nombre de Secretaría de Salubridad y Asistencia, por otro lado se funda institucionalmente la seguridad social y se crea el primer Instituto Nacional de Salud, hoy Hospital Infantil de México. Posteriormente en 1944 se creó el Instituto Nacional de Cardiología, en 1946 de cancerología y en 1960 el de neurología (Frenk, 2014)2.

En el mismo año surge la creación del Instituto Mexicano del Seguro Social, pilar de nuestro actual sistema de salud, se crea con la finalidad de brindar servicios de salud de primer, segundo y tercer nivel a las personas que hacen parte de la economía formal del país, es decir, que sean asalariadas y coticen cierta cantidad de manera anual. Posteriormente, en 1950 surge como réplica de este el Instituto de Seguridad y Servicios Sociales de los Trabajadores del Estado, la mayor diferencia es que en el primero el patrón es un particular, mientras que en el segundo es el mismo Estado. Otros prestadores de servicios de salud con la misma estructura son Pemex, Semar y Sedena, los cuales se ofrecen de igual manera para los trabajadores de estas dependencias estatales.

2 Entrevista realizada al ex secretario de Salud Julio Frenk Mora en el programa México Social emitido por Canal Once el 15/07/2014. 
La política en salud adoptada por el Estado mexicano respondía de cierta manera a la realidad social del país en aquellos tiempos, sin embargo, es aquí donde tiene lugar la segmentación en cuanto a la prestación de servicios en nuestro país y el derecho a la protección de la salud no se concibe como un bien inherente a la persona, sino como un beneficio del sector asalariado de la sociedad. Así mismo se puede apreciar que el Estado mexicano destinaba los recursos de manera desigual, inequitativa y regresivamente, tomando en cuenta grupos de ingreso, la forma de cotización de los trabajadores tenía ciertas disparidades y se propiciaba la evasión de la obligación por parte de los patrones. Se hizo completamente a un lado a la población no asalariada mediante el argumento de que los recursos no podían financiar el subsidio para todos, la realidad en muchos casos es que los auto empleadores ganaban más que un asalariado, lo que de cierta manera hubiera resultado benéfico para el sistema de salud la inclusión de este sector (Dávila \& Guijarro, 2000).

La distribución de los recursos estatales también era evidentemente desigual e injusta, debido a que las cuotas gubernamentales encaminadas al financiamiento de las instituciones de Salud provenían de los impuestos generales, lo que significaba que el mexicano no asalariado contribuía al sostenimiento del sistema de salud, pero no tenía acceso a este. De acuerdo con el ex secretario Salomón Chertorivski (Chertorivski, 2012) se realizaba una erogación hacia un asalariado en relación con un no asalariado en una proporcionalidad de 4 a 1, es decir 4 veces más recursos para el sector asalariado. Con lo anteriormente descrito se conforma la primera generación de los sistemas de salud, encaminado a la protección y desarrollo industrial mediante el otorgamiento de beneficios a la clase obrera. La Secretaría de Salud por otro lado se encargaba de la provisión de los servicios a la clase no asalariada, a través de cuotas de recuperación que en la mayoría de las ocasiones representaban gastos catastróficos para el sector de la sociedad de escasos recursos.

A raíz de los cambios sociales y económicos en la sociedad mexicana, el modelo laboral del sistema de salud no respondía a las exigencias, durante los años sesenta era prácticamente insostenible y la cantidad de personas sin acceso a la salud era una gran proporción de la población, durante la década de los setenta se desarrolla la segunda generación de reformas en el Sistema de Salud y se crean programas como IMSS-Coplamar (1979) que luego se renombra como IMSS-oportunidades (1989) con la finalidad de brindar servicios de salud en comunidades rurales y de extrema pobreza.

En 1983 se reconoce el derecho a la protección de la salud como derecho humano en el ordenamiento constitucional mexicano. Se expide en 1984 la Ley General de Salud, la cual, según lo establecido en el texto constitucional, regularía la forma y el acceso a los servicios de salud, además de plasmar el funcionamiento del sistema nacional de salud, se definen ciertos parámetros de salud y los servicios por prestar en relación al derecho. Un punto importante para contemplar es que en México se permite la inclusión del sector privado en la prestación de servicios de salud, la regulación de estos prestadores es a través de la ley antes mencionada, sin embargo, 
se limita únicamente al cumplimiento de los parámetros sanitarios de funcionamiento y no a la regulación de la calidad de los servicios.

La atención de la implementación de las políticas públicas se centra en la periferia de las problemáticas, en vez de reforzar acciones preventivas se encaminan los esfuerzos gubernamentales hacia una atención primaria que también deja mucho que desear. Se ha enfocado toda la atención a la asistencia médica en su carácter individual y la función de salud pública ha quedado rezagada a pesar de representar un factor determinante de la salud en su aspecto colectivo (Tapia \& Motta, 2005).

Por otro lado, en cuanto a desarrollo social el Estado mexicano falló en la regulación de otras áreas indispensables y factores condicionantes de un óptimo estado de salud, empezando por los índices de pobreza en el país, el desempleo, garantizar los insumos básicos para la salud, tales como adecuada alimentación, nutrición, educación, vivienda, en fin, se necesitaban redirigir los esfuerzos gubernamentales e implementar una adecuada política social de manera que la inversión de recursos fuera cualitativa y no cuantitativa para lograr la finalidad, Dávila y Guijarro menciona que "toda política social es una política de salud" (Dávila \& Guijarro, 2000).

En seguida tenemos la debilidad institucional, no solo en cuanto a calidad de atención médica sino también a la evidente falta de recursos humanos en el sector, México es uno de los países que más presupuesto destina a trabajadores administrativos, esto generó una precarización laboral para las personas encargadas de la salud, lo cual, a su vez, contribuyó a una problemática presente no solo en nuestro país si no en América Latina en general, el desplazamiento de personal médico (Salud, 2012).

\section{0-2000}

En vista de las múltiples problemáticas, la cantidad de personas sin acceso a los servicios de salud, la debilidad institucional, la falta de regulación y control de calidad en la prestación de servicios de la salud, además de la incorrecta distribución de los recursos financieros y la poca inversión en salud por parte del estado mexicano en relación con el PIB, en 1997 se da el paso a la descentralización de los servicios de salud. De esta manera se delegan ciertas responsabilidades y facultades a las entidades federativas y surgen las Secretarías de Salud del Estado (SESA) como otra parte de las instituciones del sector público, así mismo, las instituciones como IMSS e ISSSTE crean sucursales estatales para la prestación de servicios. En ciertos casos la delegación de las facultades se atribuyó también a los municipios.

La descentralización sucede como respuesta a la sugerencia de organizaciones internacionales, tales como: el Banco Mundial y el Fondo Monetario Internacional. La justificación yacía en que un sistema en donde se promueva la participación democrática de la ciudadanía desde los niveles locales equivaldría a una mayor exigencia y control de la calidad de los servicios. Se dotó a las SESAS, instituciones de Seguridad Social Estatales de plena autonomía en cuanto a funcionamiento y regulación de los recursos destinados por la federación. Se reformó la Ley de Coordinación Fiscal a efecto de 
implementar un Fondo de Aportaciones para los Servicios de Salud. Los parámetros utilizados para la asignación de recursos resultaban inequitativos, pues se basaban en tasas de mortalidad e implementación de infraestructura, lo cual resulta inadecuado para medir la verdadera capacidad y calidad de una entidad federativa en cuanto al cumplimiento de sus obligaciones. Lo que sucedió es que se propició una mayor fragmentación del sistema, la desorganización de los servicios estatales en cuanto a control de pacientes y utilización de recursos dio lugar a una gran fuga de presupuesto, las cuotas de recuperación establecidas por las SESAS para la prestación de servicios a las personas no asalariadas resultaban insustentables para las economías familiares de los sectores pobres de la sociedad. La descentralización acentuó la inequidad de prestación de servicios de la salud en las entidades federativas, al tener la libertad de aplicar los recursos federales a discrecionalidad algunas entidades invertían más en nuevas tecnologías en vez de programas preventivos o bien, los estados más pobres no tenían la capacidad financiera de reclutar y calificar al personal ni para desarrollar la infraestructura adecuada para la prestación de servicios de las SESAS, en fin, hubo una mala implementación de política de administración pública y no se obtuvieron los resultados esperados (Ugalde \& Homedes, 2002).

Es en 1998 cuando se empiezan a concentrar los esfuerzos gubernamentales para extender la cobertura de los servicios de salud a las zonas marginadas, la Secretaría de Salud después de la descentralización reorienta sus funciones hacia un aspecto más normativo y vigilante relativo a la inspección de calidad e implementación de programas, de esta manera en este año se lanza el Programa Ampliación de la Cobertura el cual comienza mediante unidades móviles que ofrecían atención médica ambulatoria en zonas rurales para luego comenzar a invertir en infraestructura para la debida prestación de servicios. Es entonces que entra en vigor el programa IMSS solidaridad, el cual focaliza sus actividades en municipios rurales de los Estados con más rezago.

Hasta este periodo se puede analizar lo siguiente: el inicio de una política pública que segmenta a la sociedad, el crecimiento de un sistema de salud fragmentado de tal manera que la forma de cotización, los servicios ofrecidos, las infraestructuras y el personal médico son cuestiones completamente heterogéneas entre unos y otros. Dentro de cada institución se carece de un sistema de referencia y contra referencia, lo cual permite que se presten los servicios de manera desorganizada y genera gastos innecesarios al no tener un control de los usuarios y los servicios que estos requieren. La atención primaria y servicios de urgencias de las instituciones son deficientes. Por otro lado, el sector privado enfoca sus servicios en la cobertura de gastos médicos mayores, mediante contratos que contienen cláusulas evidentemente desventajosas para los contratantes y los servicios ofrecidos, ni siquiera garantizan la cobertura total de padecimientos. Durante este periodo es apreciable el rezago de México en parámetros mundiales de inversión al gasto público en salud con relación al PIB, siendo aproximadamente de 3.2\% (Dávila \& Guijarro, 2000). 
Para el 2000 la situación era la siguiente: un monopolio de los proveedores de los servicios de salud, no había fomento de competencia entre las instituciones. La realidad era que los recursos aún eran distribuidos de manera regresiva, beneficiando al sector de la sociedad que más ganaba, prueba de esto es el descuido y rezago de las comunidades indígenas para acceder a los servicios de salud. La infraestructura existente se localiza en zonas urbanas, por lo cual si alguna persona cuya residencia es en una zona rural y necesita de algún servicio requiere recorrer distancias largas a efecto de lograrlo.

\section{0-2012}

Durante la presidencia de Vicente Fox el entonces secretario de Salud, Julio Frenk, realizó un estudio a través del Programa Nacional de Salud acerca de las desigualdades sociales y la repercusión en el acceso a la salud (Gonzalez-Pier, Gómez-Dantés, Lezana, Knaul, \& Frenk, 2007). En el contexto global los países potencias reforman sus sistemas de salud hacia una cobertura universal, igualitaria y equitativa en los servicios de salud y la distribución correcta y justa del gasto público, en el caso de México este comienza el siglo XXI de manera rezagada. En ese año el IMSS cubría a trabajadores del sector privado y sus familias sumando un $40 \%$, en el caso del ISSTE es un 7\% y el sector privado se encargaba del 3-4\%, por lo tanto, aproximadamente un $50 \%$ de la población carecía de algún tipo de aseguramiento. Se reconoce que aunque las personas no asalariadas tienen acceso a servicios de salud altamente subsidiados y mediante tasa de cuotas de recuperación estas siguen repercutiendo de manera fatal a sus economías. En este estudio se identificaron 5 problemáticas del sistema de salud en conjunto, a saber:

1) El gasto total en salud

2) La predominancia del gasto de bolsillo

3) La injusta distribución de los recursos públicos entre los asegurados y no asegurados y entre los estados

4) La inequidad de los estados en el financiamiento hacia el cuidado de la salud

5) La falta de inversión en infraestructura para el sector

Aunado a lo anterior, en el mismo año se realiza un informe de salud a nivel mundial en donde México registró un gasto total en salud equivalente al 5.2\% con relación al PIB situado por debajo del 7\%, el cual es el estándar en América Latina, y gran parte de ese gasto corresponde a gasto de bolsillo (Gonzalez-Pier, GómezDantés, Lezana, Knaul, \& Frenk, 2007).

Por lo tanto, a pesar de que el derecho a la protección de la salud fue reconocido como derecho humano en nuestra constitución décadas atrás, en la realidad la forma de distribución de servicios de la salud era claramente poco ética y contrario a lo establecido en el Convenio de los Derechos Económicos, Sociales y Culturales de la ONU, porque la presión para una reforma en el sistema provenía no solo de la contundente necesidad de la población sino de organismos internacionales. Es así 
que se origina la reforma que da lugar al Sistema Popular de Seguridad Social, cuyo componente clave es el Seguro Popular, la fundamentación se basa en mera ética, en disminuir la desigualdad y poder garantizar a todos un acceso a la salud de manera universal, es decir, para todos y de la misma manera.

La médula de esta reforma se centra en la modificación de la Ley General de Salud, la creación de la institución previamente mencionada y una reorganización del Sistema de Salud en cuanto a la armonización institucional horizontal de sus tres funciones; rectoría, financiamiento y prestación de servicios, para con esto corregir la fragmentación y segmentación por sectores de población. Brevemente, los cambios sustanciales en cuanto a rectoría se encaminan a corregir los daños provocados por descentralización, la Secretaría de Salud mantiene un control más estricto relativo a las asignaciones presupuestales dirigidas a los Estados, se intenta garantizar en los estados un paquete básico de servicios de salud y la certificación de sus instituciones.

Durante este periodo el aumento en el presupuesto por parte del gobierno en materia de salud se elevó en un 1.5\% adicional con relación al PIB para la creación del Seguro Popular, ahora bien, la forma de financiamiento de esta institución es muy parecida al modelo del IMSS o ISSTE, la contribución social por familia es financiada en su totalidad por el gobierno federal, basado en el principio de solidaridad los gobiernos estatales junto con el federal emiten una cuota corresponsable y una aportación por parte del beneficiario que debe ser de manera progresiva y distributiva, tomando en cuenta el ingreso de cada familia, se define el gasto total menos el gasto en alimentos, el porcentaje superior es de $5 \%$, los dos quintiles más pobres de la población están exentos de todo pago (Chertorivski, 2012).

En cuanto al presupuesto para los paquetes de servicios básicos en los Estados se continua de manera descentralizada, pero a nivel federal se crean fondos para poder garantizar el acceso a la salud en todo momento, el más importante y que consiste en un $8 \%$ de la contribución social es el Fondo de Protección para gastos catastróficos, otro destinado a la infraestructura (2\%) y aquel que prevé los cambios inflacionarios y las cuotas retrasadas (1\%) (Gonzalez-Pier, Gómez-Dantés, Lezana, Knaul, \& Frenk, 2007).

A propósito de la prestación de servicios, la clave del proceso se encuentra en la definición del paquete de servicios, esto a través del estudio de evidencias que de manera contundente reflejen las necesidades de la comunidad y se dinamice conforme a los cambios epidemiológicos, además de que a la larga se amplíen los servicios contenidos en él, la incorporación de instituciones de salud que presten los servicios al seguro popular, en la que están integradas las secretarías de salud de los Estados y de manera menos prominente el sector privado (Frenk, 2014).

Se busca cambiar la política de salud pública hacia una preventiva, se lanzan programas como PrevenIMSS y PrevenISSSTE además de encaminar los esfuerzos a reforzar la atención primaria mediante la debida capacitación del personal médico, y del cambio en la manera de asignar recursos a los Estados y cómo estos los aplican, en este caso lo que se toma en cuenta es el número de afiliados al Seguro Popular, 
al ser estos la parte de la sociedad más desprotegida y no asegurada, los recursos se etiquetan hacia este fin lo cual hace que su distribución sea progresiva. Otro factor importante que pretendía la reforma en ese entonces era la homogenización de la calidad en la prestación de servicios, es decir, sea la persona afiliada al IMSS, ISSSTE, Seguro Popular o cualquier otro, no varíen los parámetros de calidad en la atención y se garantice de la misma manera la protección de la salud.

Todavía se presenta desigualdad en cuanto a inversión, acceso a los servicios públicos y calidad de salud de las comunidades indígenas, sector más desprotegido de la sociedad mexicana. Aunque en 2012 se destinó un presupuesto considerable para programas de desarrollo social $(\$ 730581,30)$ y aproximadamente un $40 \%$ de estos iban dirigidos a la salud, ni uno solo iba encaminado a mejorar las condiciones de acceso a la salud para este sector en específico. El gasto per cápita en salud es más alto para personas no indígenas, además de que en la mayoría de los casos estas tienen accesos a servicios de atención médica privada, sin embargo, es importante resaltar que aunque evidentemente hay un factor étnico en esto el problema sustancial son las barreras económicas, debido a que este sector también se encuentra dentro de los dos quintiles más pobres de la sociedad (Leyva-Flores, et al., 2014).

Instrumentos como la Encuesta Nacional de Salud y Nutrición nos permite apreciar los resultados ofrecidos por las reformas en materia de leyes y estructuras del sistema de salud, así como la situación real de las enfermedades, la calidad y percepción de los servicios de salud y los factores de nutrición y los desequilibrios en esta. En la última edición del 2012 los resultados en cuanto a cobertura universal fueron los siguientes:

\begin{tabular}{|l|c|}
\hline \multicolumn{1}{|c|}{ Institución Prestadora de Servicios } & Porcentaje de población cubierta \\
\hline IMSS & $30.42 \%$ \\
\hline ISSSTE & $3.99 \%$ \\
\hline ISSSTE estatales & $1.62 \%$ \\
\hline PEMEX & $0.40 \%$ \\
\hline SEDENA & $0.34 \%$ \\
\hline Seguro Popular & $36.55 \%$ \\
\hline Privado & $0.44 \%$ \\
\hline Otro & $0.62 \%$ \\
\hline Sin seguro & $25.43 \%$ \\
\hline
\end{tabular}

(ENSANUT, 2012).

De lo anterior es fácil discernir que en la actualidad la institución con mayor número de afiliados es el seguro popular, seguido por el IMSS y la siguiente cifra más alta es la alarmante cantidad de mexicanos que aún no cuentan con ningún tipo de aseguramiento. Si bien se ha presentado un gran desarrollo en el sistema de salud y nos encontramos en el camino correcto hacia la cobertura universal, también 
es necesario señalar las fallas persistentes del sistema, la reunificación y falta de coordinación entre instituciones persiste, la falta de homologación de parámetros de calidad y competencia, el control de insumos para la salud, la adecuada capacitación y distribución de recursos humanos, la ampliación de los servicios ofrecidos por el Seguro Popular en atención a las necesidades reales de la población.

\section{2 - actualidad}

Según estadísticas del Banco Mundial y Organización Mundial de la Salud, la situación actual en México en cuanto a inversión en sector salud oscila en $6.2 \%$ en relación con el PIB (2010-2014) a la baja, el gasto per cápita se encuentra en US\$ 664 lo cual ha representado un incremento. Ahora bien, de ese $6.2 \%$ el gasto público representa un $51.7 \%$ y el gasto privado todavía representa un $48.26 \%$. En comparación con países como Brasil (9.7\%), Costa Rica (9.9\%), Chile (7.7\%) nos encontramos todavía por debajo, tomando en cuenta que estos países tienen aproximadamente una misma capacidad de desarrollo que México (Banco Mundial, 2014) (Organización Mundial de la Salud, 2015).

En otras estadísticas el Banco Mundial registra que el gasto privado en salud de los hogares en México es de un 95.1\% en el periodo 2010-2014, lo cual representa que aunque la cantidad de personas aseguradas por el Seguro Popular representó un gran porcentaje de la población y que se redujo el gasto catastrófico en familias, los hogares siguen desembolsando por concepto de gratificaciones y pagos en especie a médicos y proveedores de fármacos, dispositivos terapéuticos y otros bienes y servicios destinados principalmente a contribuir a la restauración o la mejora del estado de salud, lo que nos remite al pago de servicios segmentado por capacidad económica (Banco Mundial, 2014).

La política pública actual en materia de salud envuelve una reforma constitucional al artículo cuarto de la Constitución, a efecto de implementar un Sistema Nacional de Salud Universal, en el que el estatus laboral de la persona es irrelevante y la concepción del derecho a la protección de la salud como derecho humano es fuertemente arraigada. El objetivo principal es asegurar a los individuos el acceso a los servicios médicos, de la mano con la reforma constitucional se necesitará la creación de una legislación para el Sistema Nacional de Salud Universal, reforma a la Ley del IMSS, del ISSSTE y a la Ley General de Salud. Además de la creación del Sistema Universal se buscará una reorganización en la estructura, funcionamiento y financiamiento del actual sistema de salud.

En la propuesta realizada a principios del sexenio por la actual Secretaria de Salud, doctora Mercedes Juan López, se pueden destacar los siguientes puntos: $(2014)^{3}$

3 Resumen de la propuesta del ejecutivo presentada por la actual secretaria de Salud Mercedes Juan en la Conferencia Internacional "Hacia la Cobertura Universal", llevada a cabo en Lima, Perú. 
- La evolución de políticas públicas en salud va orientada a un sistema universal de la misma.

- La transición epidemiológica y las enfermedades (crónico-degenerativas) que más afectan a la sociedad mexicana requieren de una respuesta rápida y eficaz.

- Salud pública (vigilancia y prevención), atención médica (de calidad y para todos) y regulación sanitaria y política fiscal en beneficio de la salud.

- Se reconoce el excesivo gasto administrativo que México realiza, ubicado en un $17 \%$ solo en el sector público.

- La consecución de un sistema de seguridad social universal, en donde una de sus cauces sea el sistema universal de salud.

- La persistente desigualdad de la prestación de servicios de salud en las entidades federativas.

En este panorama lo más destacable de la propuesta actual es la separación del apartado de salud de seguridad social, para así enfocarse a la regulación y control de la prestación de servicio. Luego entonces lo que se busca para el Sistema Universal de Salud es la unificación de los servicios a través de la integración funcional de las instituciones existentes, para así reorientar y reforzar la capacidad rectora de la Secretaría de Salud como eje principal del sistema de salud frente a las instituciones de seguridad social. Además de hacer uso eficaz de los recursos y fomentar la rendición de cuentas por parte de las autoridades.

Los cuatro ejes en los que se sostiene esta propuesta son:

1) La portabilidad como eje principal, es decir, la capacidad del individuo de decidir la institución en la cual quiere le atiendan, y que más le convenga, por ejemplo: si un derechohabiente del IMSS considera que para su padecimiento hay una atención más adecuada en el ISSSTE pueda llevar a cabo ahí su tratamiento.

2) La convergencia, es decir, el acceso equitativo y la estandarización de la calidad tanto en cuestiones técnicas como de trato.

3) La integración, homologar las instituciones en cuanto a funciones y separar la salud de la seguridad social.

4) Acceso efectivo, independientemente del estatus laboral o condición social del individuo.

Si bien el Seguro Popular hasta el 2013 cubrió un total de 285 intervenciones, catalogó que se ha ido ampliando al paso del tiempo, de acuerdo con la realidad en México se pretende priorizar las enfermedades que representan más gasto y repercusiones en la salud de los mexicanos y seguir reduciendo el impacto de gasto catastrófico en los hogares como lo ha venido haciendo el Fondo de Protección para Gastos Catastróficos (FPGC) que hasta el 2013 tuvo cobertura en un total de 59 intervenciones. Se busca definir el Paquete de Garantías Explícitas de Salud a efecto 
de hacerlo exigible como cualquier otro servicio ante un órgano competente. Luego, entonces, los paquetes que se pretenden ofrecer en el sistema universal son: de salud pública, de intervenciones de alto costo y de beneficios básicos de baja complejidad (Juan, 2014).

En cuanto a financiamiento, se pretende la creación de un Fondo para Salud pública y un Fondo del Sistema Nacional de Salud Universal lo que explícitamente requeriría que los montos recaudados por IMSS e ISSSTE y demás empresas públicas también formaran parte de este. A la larga se pretende asignar recursos por persona con base en el perfil de riesgos (Gakidou, Murray, \& Frenk, 2000). Se deja abierta la posibilidad de la participación del sector privado en la prestación de servicios a través de subsidio en casos donde se compruebe su necesaria utilidad (Juan, 2014).

A principios de sexenios la reforma se veía prometedora, actualmente la realidad es otra, y no ha tenido resultados tangibles en cuanto a la garantía del derecho a la protección de la salud. En primera instancia, la inversión en el sector como vimos al principio de este apartado sigue estando por debajo de lo logrado por otros países en la región de América Latina, según la Encuesta Nacional de Salud y Nutrición 2012 la percepción que los usuarios tienen de las instituciones públicas gira en torno al mal trato, las excesivas horas de espera y la mala atención médica, el rechazo por parte de las instituciones de seguridad social al fondo único en el sector (ENSANUT, 2012).

Según Asa Cristina Laurell (2014) ${ }^{4}$ la reforma que se quiere impulsar tiene tintes neoliberales al dejar abierta la participación del sector privado, a la implementación de estrategias de mercado para hacer competencia entre las instituciones, y a una política en salud restrictiva con referencia a los PGES debido a que la "portabilidad" se encuentra limitada solo a ciertas enfermedades. Afirma que la propuesta presentada es retomada de la que anteriormente se presentó (FUNSALUD, Banco Mundial, Soberon, Frenk, Juan) y que no se encuentra basada en resultados que demuestren éxito en la implementación. Un ejemplo claro de esto es la política de descentralización de los servicios de salud que únicamente fragmentó más el sistema y no tuvo resultados positivos.

Concordamos con que el número de afiliados no es un reflejo efectivo de que todas esas personas tengan acceso a los servicios de salud, la mercantilización en el sistema de salud no es un proceso que vaya a beneficiar la igualdad y equidad en el acceso a los servicios y tampoco es adecuado limitar los servicios mínimos a los que tiene derechos las personas mediante los Paquetes Básicos (Cruz Martinez, 2014).

La situación del país respecto a la reforma en el sector salud es crítica, el IMSS presenta un déficit financiero insostenible, la situación es de años atrás cuando se pronosticaba que justo en el 2015 los fondos que garantizaban el cumplimiento del Régimen de Jubilados y Pensionados (una de los erogaciones de mayor cuantía) entre

4 Columna publicada en el periódico La Jornada, 08/10/2014.

5 Columna publicada en el periódico La Jornada, 09/12/2014. 
otras, llegarían a su límite. En el informe más reciente el IMSS (2014-2015) manifestó que la situación económica es prácticamente insostenible y que las diversas rutas de privatización se presentan como alternativa viable, de manera implícita rechazó la idea de una unificación de fondos, cuestión que es parte importante de la reforma para la creación de un sistema de salud universal. La idea de una privatización de los servicios de salud conmocionó a la sociedad mexicana, la cual indignada ha manifestado su descontento a través de protestas ciudadanas.

La cuestión es la siguiente, se pretende que ciertos servicios que el IMSS presta a sus derechohabientes sean subrogados o subcontratados mediante "licitaciones con requisitos muy específicos" a actores del sector privado. El debate respecto a este tema es todavía poco claro debido a que los actores gubernamentales no han sido claros ni contundentes respecto a lo que realmente se pretende, la realidad siempre viene implícita entre líneas. Por otro lado, se planea una reformulación al financiamiento de los servicios de salud a través de impuestos generales lo cual podría ser un buen paso para comenzar a difuminar esa marcada desigualdad entre sector formal e informal de la economía mexicana, la reforma también sacudiría a la industria farmacéutica y se abriría el mercado para las aseguradoras privadas.

Es innegable que el actual sistema de salud no es competente para enfrentar la transición epidemiológica del país, las enfermedades que más afectan a los mexicanos representan tratamientos costosos para el Estado y es necesario una reforma estructural total, la cuestión aquí es: ¿El modelo de asociación públicaprivada es la respuesta a nuestros problemas? ¿La apertura de los servicios de salud al mercado no representaría una violación al derecho humano a la protección de la salud? Nos encontramos ante un paradigma, la actuación del sector privado en el contexto mexicano no ha traído resultados favorables en el pasado, pero la política de bienestar social del país resulta ineficaz e inadecuada para el contexto económicosocial actual, la presión por parte de las agencias financieras internacionales para implementar esta nueva política social juega un papel muy importante; sin embargo, es la debilidad institucional que caracteriza a México la cual tiene en jaque al país. Sin duda, los próximos meses serán de gran relevancia para saber qué camino tomará el Estado mexicano y que tan apegadas a la protección de la salud como derecho humano sean sus acciones.

\section{MECANISMOS DE EXIGIBILIDAD DEL DERECHO HUMANO A LA PROTECCIÓN DE LA SALUD Y LEGISLACIÓN APLICABLE}

Una vez mostrado el panorama, el derecho a la salud en México aún dista de ser universal y equitativo, la realidad es que el gobierno actual ha tenido que redefinir su propuesta en materia de salud en vista del poco crecimiento económico que ha tenido el país. Los cambios económicos poco alentadores han tenido como resultado un "recorte de presupuestos" del sector público y la aplicación de políticas de austeridad. 
En el caso del sector salud, la propuesta del ejecutivo se ha quedado paralizada e incluso habrá de estructurar una nueva estrategia que vaya de acuerdo con la realidad económica del país. Ante tal situación, el gobierno se excusa de falta de presupuesto para poder alcanzar las metas tanto en salud como en otros sectores y la promesa de un sistema universal de salud se queda en una mera expectativa.

Sin embargo, hay una cuestión que es de especial importancia, el gobierno no puede evadir sus responsabilidades, en apartados anteriores se trató del tema de los derechos sociales y su naturaleza. La satisfacción de estos derechos antepone la capacidad de desarrollo económico de un país y el disfrute de otras prerrogativas, entre estas, los tan protegidos derechos civiles y políticos cúspide del Estado de Derecho. Ahora bien, qué pasa entonces con los derechos sociales, si no se cumplen evidentemente hay repercusiones para la sociedad pero y ¿para el Estado?

Según Courtis y Abramovich (2014) la problemática de la exigibilidad de los derechos sociales yace en las siguientes cuestiones: la definición del contenido exigible de estos, anteriormente se tenía la idea de que son simples normas programáticas que el Gobierno tiene que tomar en cuenta en el desarrollo de su planeación y se disminuyen en muchas ocasiones a promesas electoreras y pactos políticos que al final no cumplen con lo propuesto (Carbonell, 2012). El contenido referente a la conducta exigible al Estado, en el caso de los derechos civiles y políticos se tiene la concepción de que el Estado debe únicamente abstenerse de no vulnerarlos, siendo esto el estandarte del Estado de Derecho; concuerdo en este caso con Courtis y Abramovich en que el Estado también pone en marcha un aparato gubernamental conocido como poder judicial a efecto de hacer justiciables y exigibles esos derechos, es el mismo Estado el que pone los límites y los protege, por lo que hay una conducta tanto de abstención como de acción. Con los derechos sociales es lo mismo, ambas conductas se manifiestan, pero en este caso el cumplimiento del derecho depende directamente de la disposición de fondos públicos, cuestiones administrativas, desarrollo de normatividad efectiva y un control judicial.

Respecto a la justiciabilidad, se refiere a la capacidad que tiene un individuo o colectividad de acudir ante un juez o tribunal y exigir el cumplimiento efectivo de un derecho. Los argumentos que respaldan la idea de que los derechos sociales no son compatibles con la justicia ordinaria, son: que no hay un derecho subjetivo inminentemente definible a reclamar, la legitimación activa respecto a que los derechos sociales representan intereses colectivos, la satisfacción urgente en cuanto a que se necesitan fondos públicos para dar cumplimiento. En comparación con los derechos civiles carecen de especificación detallada, por lo tanto, caen en una generalidad que se presta a interpretación y discreción, y por último, la emisión de sentencias dado el caso por parte del poder judicial, que obliguen al Estado a cumplir no cuentan con resguardos procesales que las hagan efectivas (Carbonell, 2008).

Respecto a lo anterior hay que partir desde el punto en que los derechos sociales son compromisos adquiridos por el Estado, contenidos en el ordenamiento máximo 
de este, así como en convenios internacionales ${ }^{6}$ que explícitamente coartan la posibilidad al Estado de escudarse de la exigibilidad de los derechos por escases de presupuesto, incompatibilidad de los recursos jurídicos existentes y, sobre todo, la de realizar políticas regresivas en la materia que limiten el disfrute de los derechos. Todo derecho posee una característica que lo hace justiciable, por tanto la cuestión aquí es eminentemente de carácter político, por lo que se requiere una transición a lo jurídico (Tapia \& Motta, 2005).

Luego entonces, el Estado tiene la obligación de satisfacer necesidades, lo cual involucra un presupuesto y autoridades políticas, cuando hay discriminación en el ejercicio del derecho hay una evidente violación de este, en el caso de México la discriminación en la obtención de servicios de salud por parte de los sectores indígenas y más pobres de la población es un problema latente, es entonces necesario establecer medidas de protección y aseguramiento que garanticen a estos sectores el derecho, es momento de pasar la transición entre la línea de lo político y lo jurídico.

En un contexto jurídico la interpretación acorde a los derechos humanos debe de ser pro homine, en el sentido de que se da una preferencia interpretativa y normativa a la disposición que amplíe la esfera de garantías de la persona, y no al contrario, este principio ha sido adoptado por el Estado mexicano mediante la reforma del 2011 al artículo primero de la Constitución, en el que se implementa el bloque de constitucionalidad conformado por la Constitución y tratados internacionales, se establece el derecho que tiene la persona a gozar de todas las prerrogativas que estos contengan y que además sean respetadas y protegidas. Los derechos sociales no son simples normas enunciativas establecidas como buenos deseos en el ordenamiento, son parámetros constitucionales plenamente exigibles y la cuestión versa bajo qué parámetros de garantismo los órganos estatales interpretan la constitución y leyes secundarias en aras de la protección del Derecho (Corte, 2013).

El derecho a la protección de la salud es un precepto constitucional de carácter prestacional, el cual consiste en la actitud del Estado de proporcionar atención médica, salud pública y asistencia social. En relación con lo ya tratado, el Estado mexicano está obligado mediante tratados internacionales y la Constitución para con sus ciudadanos de instituir garantías institucionales y normativas que permitan la disponibilidad, accesibilidad, aceptabilidad y calidad de los servicios de manera universal, además de contar con medidas apropiadas de carácter legislativo, presupuestario y judicial que lo hagan efectivo (Courtis, 2007). Siguiendo la línea de la posibilidad de exigibilidad del derecho a la salud, este puede hacerse valer frente a las autoridades políticas como a las parlamentarias. En el sentido de que en primera instancia el legislador debe resguardar el derecho y definir las obligaciones del Estado y las prerrogativas de los particulares y prevenir la vulneración mediante el establecimiento de mecanismos de protección y, por otro, las autoridades políticas

6 Opinión General No. 9 emitida por el Comité Internacional de los Derechos Económicos, Sociales y Culturales en 03/12/98. 
estatales tienen la obligación de respetarlo mediante el adecuado resguardo y uso del presupuesto dirigido al sector, además de darle cumplimiento tomando en cuenta a los grupos vulnerables y situaciones reales de un país a efecto de satisfacer a todos el acceso (Carbonell, 2008).

De acuerdo con el artículo cuarto constitucional, la legislación en la materia que regula el acceso a los servicios de salud es la Ley General de Salud, establece la coordinación entre la Federación y las entidades federativas, la estructura del Sistema Nacional de Salud y su finalidad. En la ley se mencionan como autoridades sanitarias al Presidente de la República, el Consejo de Salubridad General, la Secretaría de Salud y los gobiernos de las entidades federativas, incluyendo al del Distrito Federal. Se define la competencia y responsabilidades de cada autoridad en los diversos niveles de gobierno, en lo que respecta a salubridad general. En la ley se menciona la prioridad que deben tener los grupos vulnerables, así como las responsabilidades en cuanto a prestación de servicios de salud públicos, los cuales deben regirse por criterios de universalidad y gratuidad fundados en las condiciones socioeconómicas de los usuarios. En su artículo 71 bis se menciona la protección social a la salud y los parámetros de necesidad mediante los cuales se debe brindar el acceso efectivo, se menciona también como un mínimo a satisfacer por parte del Estado los servicios de consulta externa en el primer nivel de atención, así como de consulta externa y hospitalización para las especialidades básicas de: medicina interna, cirugía general, ginecobstetricia, pediatría y geriatría, en el segundo nivel de atención (establecimiento de un decent mínimum health care) (Courtis, 2007).

En relación con el Sistema de Protección Social también se definen los parámetros para la certificación de calidad de las dependencias que presten los servicios de salud, las cuotas de recuperación y los derechos y obligaciones de los beneficiarios, pero no hay que dejar de lado las obligaciones en materia de salud pública, la coordinación fiscal entre las instituciones, la regulación de los insumos básicos que son actividades estatales que afectan directa o indirectamente a las personas en el goce del derecho, en lo que respecta a la exigibilidad del derecho a la protección de la salud la ley hace mención del recurso de revocación de disposiciones administrativas, sin embargo, no es contundente ni clara en lo que respecta a la protección de la salud como derecho humano y los procedimientos que establece para la reclamación de los derechos de los usuarios de salud (quejas), en muchas ocasiones se atoran en las barreras administrativas. Otras disposiciones normativas, tales como los reglamentos y las controvertidas normas oficiales generan una sobrerregulación en algunos casos y una generalidad que se presta a la discrecionalidad administrativa, lo cual, en ciertos casos, complica aún más la definición del derecho subjetivo que se pretenda hacer reclamable.

El juicio de amparo es nuestra institución jurídica máxima cuando se habla de protección de garantías individuales, juicio mediante el cual se le impone una obligación al Estado originada de un derecho reconocido en el ordenamiento estatal o internacional y que es violentado por actos de autoridad o bien por leyes 
inconstitucionales. Aunque jurídicamente se han logrado conquistas en la extensión de la protección del juicio de amparo a los gobernados, tales como el interés legítimo individual o colectivo que deja de lado la rigurosidad de la antigua concepción del interés jurídico e individualista, la cual acentuaba los problemas de legitimación activa, la posibilidad de promover un amparo colectivo más allá de la materia tributaria y la superación de la "cláusula Otero", sin embargo, no ha tenido el alcance deseado en materia de protección a la salud, medio ambiente, desarrollo, calidad de vida, entre otros aspectos.

La Suprema Corte de Justicia de la Nación ha transitado por varias facetas de interpretación del derecho, en un principio se tomó como un simple parámetro de elaboración de políticas públicas y, por lo tanto, no exigible por constituir meras expectativas, posteriormente mediante tesis aislada XIX/2000 se le otorga el amparo a una persona a la cual se le habían negado los medicamentos contenidos en el cuadro básico para el tratamiento de VIH, esto, en virtud de que en la Ley General de Salud el acceso a los medicamentos básicos era una garantía instituida en el ordenamiento y exigible debido a una evidente necesidad de los mismos, independientemente de los factores ajenos a la responsabilidad de la persona que concurrieran (Suprema Corte de Justicia de la Nacion, 2000).

En amparo en revisión 378/2014 la Segunda Sala de la Suprema Corte resuelve en relación con el contenido y alcance del derecho humano en su vertiente de tratamiento de enfermedades y creación de condiciones que aseguren a todos la atención médica y servicios médicos en caso de enfermedad, los quejosos reclaman la omisión de llevar a cabo el proyecto "Construcción y Equipamiento de servicio clínico para pacientes con VIH/SIDA y co-infección por enfermedades de transmisión aérea" en el Instituto Nacional de Enfermedades Respiratorias Ismael Cosío Villegas, el cual consistía en la construcción de un pabellón que permitiría el no contagio de los enfermos de VIH/SIDA, quienes habían tenido repercusiones en su salud al estar en contacto con otros pacientes, lo cual representaba un riesgo constante en su salud, y el cumplimiento del proyecto en cuestión requería de una asignación de recursos que fue negada. Tanto en primera como en segunda instancia se determinó que no había violación alguna por parte del Estado y se sobreseyó el juicio. Posteriormente, la Segunda Sala realizó un control de constitucionalidad basándose en un análisis de la legislación internacional en la materia, el marco general del VIH/SIDA de acuerdo con la OMS y los parámetros necesarios para atender a los pacientes, la legislación interna y la realidad de los hechos, resolvió revocar la sentencia inicial y amparar a los quejosos, lo cual demuestra que es posible condenar a la autoridad de manera racional a efectuar conductas que conlleven la erogación de presupuesto público. Sin duda, un importante criterio en materia de derechos sociales emitido por el órgano judicial supremo considera que aún se debe admitir que la jurisprudencia en materia de derechos sociales en nuestro país ha sido precaria, las tesis aisladas solo sirven como guías interpretativas del órgano jurisdiccional, más no tienen obligatoriedad (Amparo en Revision 378/2014, 2014). 
En cuanto al cumplimiento de la obligación, es necesario establecer respaldos procesales y jurídicos a disposición del individuo o colectividad durante la ejecución de sentencia. La sentencia pronunciada en contra del Estado por incumplimiento de obligaciones mínimas de derechos sociales debe ser racional en cuanto a las posibilidades reales, si bien no se obliga al Estado a efectuar un desembolso de manera inmediata "se le pone en mora" (Courtis \& Abramovich, 2014) y se le exige el cumplimiento futuro del derecho a efecto de propiciar un logro progresivo y esto de ninguna manera significa que se priva de un contenido propio de las obligaciones de un Estado, si no que significa la imposición de un deber concreto de avanzar en el cumplimiento lo más eficazmente posible (Courtis, 2007).

En cuanto a la acción colectiva, esta va encaminada hacia lo protección de intereses difusos, individuales o colectivos, pero que trasciendan la esfera individual, puede ser presentada por medio de un representante con legitimación colectiva, es decir, para proteger el derecho de un grupo de personas, tiene su fundamento en el artículo 17 constitucional. El uso de este instrumento jurídico tiene trascendencia en cuanto a nuestro tema en la protección al derecho de la salud para reclamar daños ambientales que se le puedan ocasionar a una colectividad. Es una manera más fácil y expedita de acceder a la justicia y sin duda ayuda a la no saturación del aparato judicial, la falta de tradición jurídica y uso ha retrasado el desarrollo de este instrumento que ha tenido resultados positivos en otros países de América Latina como Brasil, Colombia, entre otros (Pisarello, 2009).

Otro instrumento jurídico es la queja ante la Comisión Nacional de Derechos Humanos contra actos de autoridades administrativas del poder federal que violenten la normatividad, ocasionen violaciones a los derechos humanos o la negativa al ejercicio de atribuciones de manera arbitraria, que resulte en conductas que atenten contra la integridad física de las personas (Moctezuma, 2000). La crítica hacia la actuación de la Comisión ha sido constante, esta tiene la capacidad de investigar presuntas violaciones y de considerar que hay suficientes elementos para probar dicha violación para entonces emitir una recomendación que en muchos de los casos no trasciende a más.

En cuanto a los derechos de los pacientes en relación con la atención médica, por vía civil es exigible siempre y cuando la relación haya surgido mediante un acuerdo de voluntades donde lo pactado hayan sido los servicios médicos en cuestión, prestados por particulares, otros órganos competentes para conocer de inconformidades es la CONAMED y la PROFECO, mediante instancias de resolución alterna de conflictos que busca dirimir controversia (Moctezuma, 2000).

La política internacional del Estado mexicano en cuanto a la instrumentación jurídica de la justiciabilidad y exigibilidad de los derechos sociales tampoco ha sido contundente. Como anteriormente se hizo mención, México es Estado parte del Pacto Internacional de los Derechos Económicos, Sociales y Culturales de la Organización de Naciones Unidas. En 2009 se lanzó la convocatoria para la firma y ratificación del protocolo facultativo del pacto en cuestión, el cual permitiría que 
las personas cuyos derechos sociales sean violentados tengan acceso a la justicia internacional ante el Comité de Derechos Económicos, Sociales y Culturales de la ONU. A pesar de que México se mostraba en un principio a favor del protocolo, este ya lleva dos años de haber entrado en vigor y México ni siquiera lo ha firmado, a pesar de que se han emitido diversas recomendaciones internacionales por parte de Bosnia, Herzegovina, Portugal y organizaciones no gubernamentales.

\section{CONCLUSIONES}

En realidad la concepción del derecho a la protección de la salud en nuestro país sí ha evolucionado de manera progresiva, los cambios globales han influido en nuestro orden jurídico y social. Sin embargo, la transición hacia la realidad ha sido lenta, nuestro sistema de salud dista de ser universal, los parámetros de calidad aun requieren de atención por parte del Estado, además que los determinantes sociales y económicos siguen siendo predominantes para el efectivo acceso a los servicios de salud.

El Estado mexicano tiene una obligación para con sus ciudadanos, el derecho a la protección de la salud es un derecho humano y parámetro de constitucionalidad, por lo tanto la actuación del Estado debe ir encaminada y racionalizada al cumplimiento de las necesidades de las personas. La delimitación de paquetes básicos de servicios de salud representa un principio de progresividad en el cumplimiento de la obligación, sin embargo, es importante que se atienda la realidad epidemiológica del país a efecto de que ese paquete resulte enunciativo y no limitativo.

La participación ciudadana se debe imponer como instrumento previo de exigibilidad al Estado del cumplimiento de sus obligaciones, en México, lamentablemente la falta de información y educación impide una sociedad informada capaz de exigir sus derechos. Por otro lado, la instrumentación jurídica para la justiciabilidad existe, sin embargo, no todos los métodos son tan efectivos como quisiéramos y la falta de tradición jurídica o desconocimiento de los mismos relegan su uso, por lo tanto nuestro medio más efectivo como ciudadanos es el juicio de amparo, la saturación de los órganos judiciales y el tiempo promedio de resolución podrían representar una dificultad en cuanto a la urgencia de las necesidades, sobre todo en materia de salud. Por último, la finalidad de los instrumentos de justiciabilidad es precisamente para impartir justicia y no para tratar de corregir fallas del sistema. México aún tiene camino por recorrer, es un país en desarrollo y por ello como Estado debe proteger aquellos factores que garantizan su crecimiento y desarrollo económico, social y cultural.

Por un lado, la política internacional e interna mexicana se apega al principio de universalidad y a la concepción de la protección a la salud como derecho que todo ciudadano humano tiene para reclamar al Estado, pero la realidad es otra, la desigualdad por sectores, la calidad mínima de los servicios de salud, la disparidad en calidad de las diversas instituciones públicas y las actuales limitaciones de un 
sistema de salud rebasado por sus obligaciones ponen al Estado mexicano en evidencia respecto al incumplimiento de su política social. Sin embargo, serán las próximas medidas que se tomen las que definirán a ciencia cierta la postura tomada respecto a la salud, para lo cual un análisis riguroso del contexto económico, político y social será necesario para implementar un régimen social que si bien al principio nos puede parecer radical tenga su justificación en la eficacia y adecuación a nuestra realidad.

\section{REFERENCIAS}

Baldwin, P. (1990). Recuperado de https://books.google.com.mx/books?hl=es\&lr=\&i $\mathrm{d}=$ KjVSn1lyurIC\&oi $=$ fnd\&pg $=$ PR $9 \& d q=$ welfare + state + peter + baldwin\&ots $=2$ ODE8dxMvY\&sig=7kg781Pkt5v57iasz4kiETn-FYI\#v=onepage \&q=welfare\%20state $\% 20$ peter $\% 20$ baldwin\& $\mathrm{f}=$ false

Banco Mundial. (2014). Recuperado de http://datos.bancomundial.org/indicador/ SH.XPD.TOTL.ZS

Carbonell, M. (2008). Recuperado de http://www.scielo.cl/scielo.php?pid=s0718$52002008000100003 \&$ script $=$ sci_arttext

Carbonell, M. (2012). La Constitución en serio. Multiculturalismo, igualdad y derechos sociales. México: Porrúa.

Chertorivski, S. (28 de noviembre de 2012). Politica de Salud en México. (R. R. de la Madrid, Entrevistador).

Corte, S. (2013). Derechos humanos: Parte general. México: Coordinacion de Compilacion y Sistematizacion de Tesis de la Suprema Corte de Justicia de la Nación.

Courtis, C. (2007). Recuperado de http://www.derechoecuador.com/images/ documentos/minj-proteccion_judicial_derechos.pdf\#page $=132$

Courtis, C., \& Abramovich, V. (28 de agosto de 2014). Recuperado de http:// repositoriocdpd.net:8080/handle/123456789/429

Cruz, A. (9 de diciembre de 2014). Un sistema universal no debe mercantilizar los servicios: experto. La Jornada.

Dantés, O., Sesma, S., Becerrila, V., Knaul, F., Arreola, H., \& Frenk, J. (enero de 2011). Recuperado de http://www.scielo.org.mx/scielo.php?script=sci_arttext\& pid=S0036-36342011000800017

Dávila, E., \& Guijarro, M. (2000). Recuperado de http://repositorio.cepal.org/ handle $/ 11362 / 5300$

ENSANUT. (2012). Recuperado de http://ensanut.insp.mx/doctos/FactSheet ResultadosNacionales14Nov.pdf

Frenk, J. (15 de julio de 2014). México social: Hacia un sistema universal de salud. (M. L. Fuentes, Entrevistador).

Gakidou, E., Murray, C., \& Frenk, J. (enero de 2000). Recuperado de http://www. scielosp.org/scielo.php?pid=S0042-96862000000100005\&script $=$ sci_arttext 
González-Pier, E., Gómez-Dantés, O., Lezana, M. A., Knaul, F. M., \& Frenk, J. (enero de 2007). Recuperado de http://scielo.unam.mx/scielo.php?pid=S003636342007000700007\&script=sci_arttext

Juan, M. (25 de febrero de 2014). Recuperado de http://www.paho.org/ forocoberturagt2014/wp-content/uploads/2014/08/Sistema-Nacional-de-SaludUniversal-experiencia-en-Mexico.pdf

Laporta San Miguel, F. (1987). Recuperado de rua.ua.es

Laurell, A. C. (8 de octubre de 2014). El sistema universal de salud panista; avanza la privatizacion del sector. La Jornada.

Leyva-Flores, R., Servan-Mori, E., Infante-Xibille, C., Pelcastre-Villafuerte, B., \& González, T. (6 de agosto de 2014). Recuperado de http://journals.plos.org/ plosone/article?id=10.1371/journal.pone.0102781

Lusitg, N. (julio de 2008). Recuperado de http://terceridad.net/PyPS/Por temas/15 Ciclo-PP concepto/Apoyo 1/Lustig\%20Nora,\%20Pol\%EDcas\%20 P\%FAblicas\%20y\%20Salud\%20en\%20M\%E9xico.pdf

Mayer-Serra, C. (marzo de 2007). Recuperado de http://www.scielo.org.mx/scielo. php?pid=S0036-36342007000200010\&script=sci_arttext

Moctezuma, G. (2000). Derechos de los usuarios de los servicios de salud. México: Instituto de Investigaciones Jurídicas de la UNAM.

Oficina del Alto Comisionado de las Naciones Unidas. (2010). Recuperado de http://www.hchr.org.mx/index.php/publicaciones/148-politicas-publicas-ypresupuestos-con-perspectiva-de-derechos-humanos-manual-operativo-paraservidoras-y-servidores-publicos-2010

Organización Mundial de la Salud. (2012). Recuperado de http://www.paho.org/ saludenlasamericas/index.php?lang=es

Organización Mundial de la Salud. (2015). Recuperado de http://apps.who.int/nha/ database/ViewData/Indicators/en

Peces-Barba, G. (1987). Recuperado de http://hdl.handle.net/10016/10462

Pisarello, G. (2009). Recuperado de http://www.justicia.gob.ec/wp-content/uploads/ downloads/2012/07/8_Proteccion_judicial.pdf\#page $=46$

Suprema Corte de Justicia de la Nacion. (2000). Tesis XIX/2000.

Suprema Corte de Justicia, Segunda Sala. (2014). Amparo en Revision 378/2014.

Tapia, R., \& Motta, M. (2005). El derecho a la proteccion de la salud pública. En I. Bresna. Memoria del Congreso Internacional de Culturas y Sistemas Jurídicos Comparados. Salud y Derecho. México: Instituto de Investigaciones Jurídicas UNAM.

Ugalde, A., \& Homedes, N. (febrero, 2002). Recuperado de http://scielo.isciii.es/ scielo.php?script $=$ sci_arttext\&pid=S0213-91112002000100004 